\title{
On the Generic Type of the Free Group*
}

\author{
Rizos Sklinos
}

\begin{abstract}
We answer a question raised in $\mathrm{Pil09}$, that is whether the infinite weight of the generic type of the free group is witnessed in $F_{\omega}$. We also prove that the set of primitive elements in finite rank free groups is not uniformly definable. As a corollary, we observe that the generic type over the empty set is not isolated. Finally, we show that uncountable free groups are not $\aleph_{1}$-homogeneous.
\end{abstract}

\section{Introduction}

As pointed out in Pil08, the free group is connected and thus it has a unique generic type over any set of parameters. In particular, there is a unique generic type over the empty set, which we denote as $p_{0}$. Furthermore, $p_{0}$ has some nice (or not so nice) properties. In [Pil09], it was shown that $p_{0}$ has infinite weight. On the way to proving this, it was also shown that the realizations in $F_{n}$ of $p_{0}$ are exactly the primitives.

In this paper we mainly explore basic model theoretic properties of $p_{0}$ and from them we deduce some useful facts about the free group. In the remainder of this section, we give some quick background, and definitions of basic notions around the free groups. In section 2, we prove the non uniform definability of the primitives in the finite rank free groups, and deduce non isolation of $p_{0}$ and other corollaries. Finally, in section 3 , we answer some questions raised by Pillay in [Pil09], including whether the infinite weight of $p_{0}$ is witnessed in $F_{\omega}$. We also show that $F_{\kappa}$, for $\kappa>\omega$, is not $\aleph_{1}$-homogeneous (as a structure).

We will freely use notions from stability theory, such as forking, independent sequence, weight, etc. Also notions from stable group theory, such as generic type, generic set, connected group, etc. Our main reference for stability is [Pil96]. Stable groups are studied elegantly, but still in great depth in Poi01. For the unfamiliar reader, there is a quick, dense introduction in Pil08, Pil09.

Finally, I would like to thank Zlil Sela and Julia Knight. Zlil Sela for a useful discussion we had at a conference in Southampton, and Julia Knight for bringing to my attention paper MV03 on the references, thus the Whitehead graph technique. Special thanks go to my thesis supervisor, Anand Pillay, for his constant help in both the preparation and the subject matter of this paper.

\section{$1.1 \quad$ Free Groups}

Let us now give some basic facts about the free groups. Let $F_{n}$ denote the free group on $n$ generators. If $E=\left\{e_{1}, \ldots, e_{n}\right\}$ is the set of generators, we can identify $F_{n}$ with the set of

\footnotetext{
${ }^{*}$ The copyright is held by the Association for Symbolic logic
} 
reduced words in $E \cup E^{-1}$, and group operation concatenation followed by reductions (such that the result is a reduced word). A word $w=u_{1} u_{2} \ldots u_{k}$, with $u_{i} \in E \cup E^{-1}$ is reduced if for all $1 \leq i \leq k-1, u_{i} \neq u_{i+1}^{-1}$. Moreover, $w$ is cyclically reduced if it is reduced and $u_{1} \neq u_{k}^{-1}$. An element of $F_{n}$ is called primitive if it belongs to some basis of $F_{n}$. It is quite clear that primitive elements form a single orbit under $\operatorname{Aut}\left(F_{n}\right)$. In [Pil09] the following fact was observed.

Fact 1.1: Let $\left\{e_{1}, \ldots, e_{n}\right\}$ be a basis of $F_{n}$. Let $m \leq n$, and $k_{1}, \ldots, k_{m}$ integers $>1$. Then $e_{1}^{k_{1}} \ldots e_{m}^{k_{m}}$ is not a primitive of $F_{n}$.

Proof. (Sketch) Aut $\left(F_{n}\right)$ is generated by Whitehead automorphisms, and no Whitehead automorphism can reduce the length of $w=e_{1}^{k_{1}} \ldots e_{m}^{k_{m}}$. Thus, $w$ and $e_{1}$ cannot be in the same orbit under $\operatorname{Aut}\left(F_{n}\right)$.

For completeness, we give the definition of a Whitehead automorphism, as given in [LS77, p.31].

Definition 1.2: Let $F$ be a free group generated by $X$, then $\tau$ is a Whitehead automorphism of $F$ if it is an automorphism of one of the following two kinds

1. $\tau$ permutes the elements of $X^{ \pm 1}$

2. for some fixed "multiplier" $a \in X^{ \pm 1}, \tau$ carries each of the elements $x \in X^{ \pm 1}$ into one of $x, x a, a^{-1} x$, or $a^{-1} x a$.

We note that in the definition above we require that $\tau$ is an automorphism. Not all maps satisfying (1) or (2) are automorphisms.

Tarski around 1945 posed the following question, "Do the free groups in more than two generators have the same common theory?"

In [Sel06] Sela proved.

Theorem 1.3 (Sela): If $2 \leq m \leq n$ then the natural embedding of $F_{m}$ to $F_{n}$ is an elementary embedding.

As this answers Tarski's question, we are now free to denote the theory of the free group as $T_{f g}$.

Sela has also proved in Sel] the following rather astonishing result.

Theorem 1.4 (Sela): $T_{f g}$ is stable.

Let us also remark here that, from previous work of Poizat Poi83, $T_{f g}$ is not superstable.

\section{Non isolation of the generic type}

Our aim in this section is to prove that the primitives are not uniformly definable in finite rank free groups. The non isolation of $p_{0}$ would only be an easy corollary. A basic step, on the way to proving this, is to show that the set of non primitives, in any free group, is "big". In the case of a definable set, this simply means generic. 
For the benefit of the reader we explain a few things about the model theoretic point of view on groups.

A group, $(G, \cdot)$, in the sense of model theory is a structure equipped with a group operation, but possibly also with some additional relations and functions. Even when we do not have explicitly any additional relations or functions, all the definable subsets, $X \subseteq G^{n}$, of the group under consideration will be part of our structure. In the case that all additional structure is definable by multiplication alone, we speak of a pure group.

We define a stable group to be a group definable in a stable theory. By this we mean that $(G, \cdot)$ is definable in a model $M$ of the stable theory $T$, and it may be equipped with some or all of the structure induced from $M$. The simplest case will be when the group coincides with the ambient structure and the underlying language is the language of groups. Indeed, this is the case for non abelian free groups.

Definition 2.1: Let $G$ be a stable group. Let $X$ be a definable subset of $G$. Then $X$ is left (right) generic if finitely many left (right) translates of $X$, by elements of $G$, cover $G$.

As for a definable $X \subseteq G, X$ is left generic iff $X$ is right generic, we simply say generic.

Definition 2.2: Let $G$ be a group. Then $G$ is connected if there is no definable proper subgroup of finite index.

Let us note here that connectedness passes to elementarily equivalent groups, so with an abuse of language we can say that $T_{f g}$ is connected, meaning that all models of $T_{f g}$ are connected groups.

Definition 2.3: Let $G$ be a stable group. Let $g \in G$, and $A$ a set of parameters from $G$. Then $t p(g / A)$ is a generic type if every formula in $t p(g / A)$ is generic.

We recall some useful facts about genericity and connectedness.

Fact 2.4: Let $G$ be a stable group. Let $X, Y$ definable subsets of $G$. Then

(i) if $X \cup Y$ is generic, then one of $X, Y$ is generic.

(ii) $G$ is connected iff there is no definable $X \subseteq G$ such that both $X$ and $G \backslash X$ are generic.

(iii) $G$ is connected iff there is over any set of parameters a unique generic type of an element of $G$.

The next important notion is that of a Whitehead graph.

Definition 2.5: Let $a=u_{1} \ldots u_{k}$, a word in $F_{n}=\left\langle e_{1}, \ldots, e_{n}\right\rangle$. The Whitehead Graph of a, $W_{a}$, is the graph with set of vertices, $V\left(W_{a}\right)=\left\{e_{1}, \ldots, e_{n}, e_{1}^{-1}, \ldots, e_{n}^{-1}\right\}$, and edges joining $u_{1}$ to $u_{2}^{-1}, u_{2}$ to $u_{3}^{-1}, \ldots, u_{k-1}$ to $u_{k}^{-1}$, and $u_{k}$ to $u_{1}^{-1}$.

We note here that the number of edges of $W_{a}$ equals the length of $a$.

Definition 2.6: Let $G$ be a graph. Then $G$ has a cut vertex, if there is a vertex, $u$, such that removing $u$ and its adjacent edges leaves the graph disconnected.

We now give some examples of Whitehead graphs in $F_{2}=\left\langle e_{1}, e_{2}\right\rangle$. The first two graphs have cut vertices, while the third does not. Also note that in the first example the graph is already not connected. 


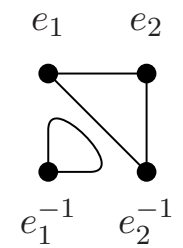

Figure 1: $e_{1} e_{2}^{2} e_{1}^{-1}$

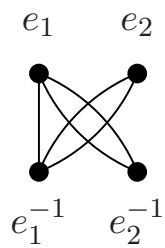

Figure 2: $\left(e_{1} e_{2}\right)^{2} e_{1}$

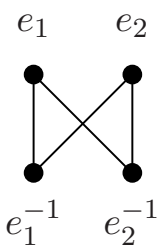

Figure 3: $e_{1} e_{2}^{2} e_{1}$

The following proposition is a weaker reformulation of Theorem 2.4 in Sta99] (see also [MV03, Theorem 6]) .

Proposition 2.7: Let $F=F_{n}$, for some $n \geq 2$. Let a be a cyclically reduced primitive of $F$. Then $W_{a}$ has a cut vertex.

The next lemma will help us prove that the set of non primitives is "big" in any free group.

Lemma 2.8: Let $F_{n}=\left\langle e_{1}, \ldots, e_{n}\right\rangle$, for some $n \geq 2$. There is a finite collection of words $\left\{w_{i j}: i, j \leq n\right\} \subset F_{n}$, such that for any $a \in F_{n}, w_{i j} \cdot a$ is non primitive for some $i, j \leq n$.

Proof. We will give an explicit description of the $w_{i j}$ 's. Let $w$ be the following word $e_{1}^{2} e_{n}^{2} e_{1} e_{2}^{-1} e_{1}$ $e_{2} e_{3}^{-1} e_{2} \ldots e_{n-1} e_{n}^{-1} e_{n-1}$. In pictures, the following graph will be part of the Whitehead graph of $w$.

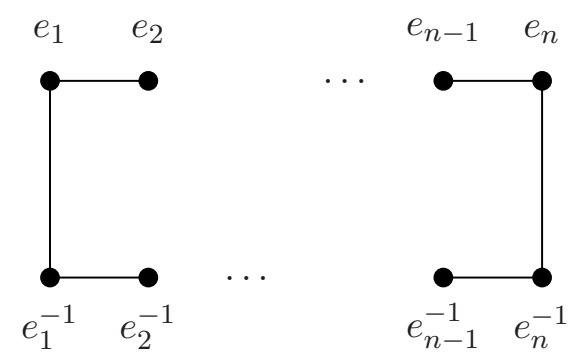

In addition, for each $p, q \leq n$, we define $w_{p q}$ to be the word $e_{p} w e_{q}$. In total we have $n^{2}$ such words. We show that these words are the $w_{i j}$ 's we wanted.

Let $a \in F_{n}$, such that $a$ starts with $e_{l}^{k}$ and ends with $e_{r}^{m}$, for some $k, m \in\{1,-1\}$ and $l, r \leq n$. Then we can choose $w_{i j}$, such that $i \neq r$ and $j \neq l$. So $w_{i j} \cdot a$ is cyclically reduced. As there is no cancellation between $w_{i j}$ and $a, W_{w_{i j}}$ contains the circle pictured above. Thus, $W_{w_{i j} a}$ does not have a cut vertex. And, by Proposition 2.7 $w_{i j} a$ is non primitive.

The only case left is when $a=1$, the identity element. By the argument above, each $w_{i j}$ is non primitive. Thus, $w_{11} \cdot 1$ is non primitive, and this completes the proof.

The following proposition is an easy consequence of Lemma 2.8 ,

Proposition 2.9: Let $F=F_{n}$, for some $n \geq 2$. Then finitely many translates of the set of non primitives, $N$, cover $F$.

Proof. Let $a \in F$. Then, by Lemma 2.8, for some $i, j \leq n, w_{i j} a \in N$. Thus, $\bigcup_{i, j \leq n} w_{i j}^{-1} N$ covers $F$. 
We next show that primitives are not uniformly definable.

Proposition 2.10: Let $F_{n}=\left\langle e_{1}, \ldots, e_{n}\right\rangle$, for some $n \geq 2$. Then there is no formula, $\phi(x, \bar{y})$, and a set of parameters $\bar{b}_{n}$ in $F_{n}$, such that $\phi\left(x, \bar{b}_{n}\right)$ defines the set of primitives in $F_{n}$, for each $n \geq 2$.

Proof. Suppose not, and $\phi(x, \bar{y}),\left\{\bar{b}_{2}, \bar{b}_{3}, \ldots, \bar{b}_{n}, \ldots\right\}$ witness it. We show that $\phi(x, \bar{y})$ has the order property in $F_{\omega}$, contradicting directly stability of $T_{f g}$.

Claim. Let $F_{\omega}=\left\langle e_{i}: i<\omega\right\rangle$. Then $F_{\omega} \models \phi\left(e_{i}, \bar{b}_{j}\right)$ iff $i \leq j$ (for $i, j \geq 2$ ).

Proof.

$(\Leftarrow)$ Suppose $i \leq j$. Then $F_{j} \models \phi\left(e_{i}, \bar{b}_{j}\right)$. But, $F_{j} \prec F_{\omega}$. Therefore $F_{\omega} \models \phi\left(e_{i}, \bar{b}_{j}\right)$.

$(\Rightarrow)$ Suppose $i>j$, but $F_{\omega} \models \phi\left(e_{i}, \bar{b}_{j}\right)$. We first show that $e_{i}$ is independent from $\bar{b}_{j}$ over $\emptyset$. By [Pil08, Corollary 2.7(ii)], $e_{i}$ is independent from $e_{1}, \ldots, e_{i-1}$ over $\emptyset$. Thus $e_{i}$ is independent from $\operatorname{acl}\left(e_{1}, \ldots, e_{i-1}\right)$ over $\emptyset$. But $\bar{b}_{j} \subseteq \operatorname{acl}\left(e_{1}, \ldots, e_{i-1}\right)$. Therefore $e_{i}$ is independent from $\bar{b}_{j}$ over $\emptyset$. So $t p^{F_{\omega}}\left(e_{i} / \bar{b}_{j}\right)$ is the unique generic type of $F_{\omega}$ over $\bar{b}_{j}$. And because $\phi\left(x, \bar{b}_{j}\right) \in t p^{F_{\omega}}\left(e_{i} / \bar{b}_{j}\right)$, $\phi\left(x, \bar{b}_{j}\right)$ is generic. But $\neg \phi\left(x, \bar{b}_{j}\right)$ defines the non primitives in $F_{j}$. So, by Proposition [2.9. $\neg \phi\left(x, \bar{b}_{j}\right)$ is also generic, contradicting the connectedness of $F_{\omega}$.

The next theorem is an easy corollary of Proposition 2.10.

Theorem 2.11: The generic type $p_{0}$ of $T_{f g}$, is non isolated.

Proof. Suppose, for the sake of contradiction, that $p_{0}$ is isolated and $\phi(x)$ witnesses it. Then for every $G \models T_{f g}$, we have $\phi(G)=p_{0}(G)$. So in particular, we have $\phi\left(F_{n}\right)=p_{0}\left(F_{n}\right)$, for any $n \geq 2$. But, by [Pil09, Theorem 2.1], $p_{0}\left(F_{n}\right)$ is exactly the set of primitive elements of $F_{n}$. So, $\phi(x)$ uniformly defines the primitives (without parameters), contradicting Proposition 2.10 .

At this point let us mention a result of Perin [Per08, which will play a central role in the next section, but also useful here.

Theorem 2.12 (Perin): Let $F=F_{n}$, for some $n \geq 2$. Let $G$ be an elementary substructure of $F$. Then $G$ is a free factor of $F$.

The next result was also proved by Nies Nie03 by slightly different methods. Our proof uses the omitting types theorem (see [Mar02, Theorem 4.2.3,p.125]), which we quickly recall.

Theorem 2.13 (Omitting Types Theorem): Let $\mathcal{L}$ be a countable language, $T$ an $\mathcal{L}$-theory, and $p$ a (possibly incomplete) non isolated $n$-type over $\emptyset$. Then, there is a countable $\mathcal{M} \models T$ omitting $p$.

Corollary 2.14: $T_{f g}$ does not have a prime model.

Proof. Let $\mathcal{A}$ be the prime model of $T_{f g}$, then $\mathcal{A} \prec F_{2}$. So by Theorem 2.12 $\mathcal{A} \cong F_{2}$, therefore $\mathcal{A}$ realizes $p_{0}$. But if the prime model realizes $p_{0}$, then any model realizes $p_{0}$. As $p_{0}$ is non isolated, this clearly contradicts the omitting types theorem.

Let us remark here that, by a result of Nielsen [Nie17, the set of primitives of $F_{2}$ is definable in $F_{2}$ (over a set of parameters). More precisely, Nielsen proved that two elements 
$a, b \in F_{2}=\left\langle e_{1}, e_{2}\right\rangle$, form a basis of $F_{2}$ iff $[a, b]$ is a conjugate of $\left[e_{1}, e_{2}\right]$ or a conjugate of $\left[e_{2}, e_{1}\right]$. Thus the following formula defines the primitives in $F_{2}$ :

$$
\exists z \exists y\left([x, y]=\left[e_{1}, e_{2}\right]^{z} \vee[x, y]=\left[e_{2}, e_{1}\right]^{z}\right)
$$

We now pass to the main part of the paper.

\section{Weight and Homogeneity}

Before we start we quickly recall the notion of weight. We work in a stable theory $T$, in a big saturated model $\mathbb{M}$ (what we usually call the monster model), and $A, B$ denote small subsets of $\mathbb{M}$.

Definition 3.1: The preweight of a type $p(x)=t p(a / A), \operatorname{prwt}(p)$, is the supremum of the set of cardinals $\kappa$, for which there exists an $A$-independent set $\left\{b_{i}: i<\kappa\right\}$, such that $t p\left(a / A b_{i}\right)$ forks over $A$ for all $i$.

The weight of a type $p, w t(p)$, is the supremum of $\{\operatorname{prwt}(q): q$ a non forking extension of $p\}$.

Using forking calculus one can see that, if $T$ is countable, then for any $a, A, w t(a / A) \leq \omega$. One could also distil from [Pil96, Lemma 3.9,p.166],Pil96, Proposition 3.10,p.167] that, if $w t(a / A)=\omega$, then for some $B \supseteq A$, such that $a$ is independent from $B$ over $A$, there is an infinite independent set, $\left\{b_{i}: i<\omega\right\}$, over $B$, such that $a$ forks with each $b_{i}$ over $B$.

This section builds upon the following crucial result, proved in [Pil09].

Theorem 3.2 (Pillay): The generic type $p_{0}$ of $T_{f g}$ has infinite weight.

The method was to find for every $n \geq 2$, a realization, $g$, of $p_{0}$ in $F_{n}$, and an independent set of realizations, $b_{1}, \ldots, b_{n}$, of $p_{0}$ in $F_{n}$, such that $g$ forks with each $b_{i}$. Furthermore, in [Pil09] it was observed that, by a compactness argument, one can find $a \models p_{0}$ and $\left(b_{i}: i<\omega\right)$ an independent sequence of realizations of $p_{0}$ in a model $G$, such that $a$ forks with each $b_{i}$ over the empty set. Therefore, a natural question is whether we can find such elements in $F_{\omega}$.

In the rest of the section we show that Theorem 2.12 cannot be extended to include $F_{\omega}$. Moreover, we answer in the affirmative the question mentioned above, and finally we show that $F_{\kappa}$, for $\kappa>\omega$, is not $\aleph_{1}$-homogeneous.

We now mention a fact, observed in [Pil09, Fact 1.9], that we will use through out the section.

Fact 3.3: Let $G$ be a connected stable group. Let $A=\left\{a_{i}: i \in I\right\}$ be an independent set of realizations of the generic type of $G$ over the empty set, in $G$. Let $\tau$ be one of the following maps:

(i) for some permutation $\pi$ of $I, \tau\left(a_{i}\right)=a_{\pi(i)}$ or $a_{\pi(i)}^{-1}$.

(ii) for some fixed "multiplier" $a_{i} \in A, \tau$ fixes $a_{i}$ and carries each of the elements $a_{j} \in A$ into one of $a_{j}, a_{j} a_{i}, a_{i}^{-1} a_{j}$, or $a_{i}^{-1} a_{j} a_{i}$.

Then $\tau$ is an elementary map in the sense of $G$. In particular $\left\{\tau\left(a_{i}\right): i \in I\right\}$ is an independent set of realizations of the generic type of $G$ over the empty set.

Also, the next result was proved in Pil09.

Theorem 3.4 (Pillay): Let $F=F_{n}$, for some $n \geq 2$. Then every maximal independent sequence of realizations of $p_{0}$ in $F$ is a basis of $F$. 
Let $\kappa$ be a cardinal (maybe infinite), we denote with $p_{0}^{(\kappa)}$ the type of $\kappa$ independent realizations of $p_{0}$. As $p_{0}$ is stationary this is a good definition. So, in other words, the above theorem says that if $\left(a_{1}, \ldots, a_{n}\right) \models p_{0}^{(n)}$ in $F_{n}$, then $F_{n}=\left\langle a_{1}, \ldots, a_{n}\right\rangle$. And every maximal independent set of realizations of $p_{0}$ in $F_{n}$ has cardinality $n$.

One might expect that Theorem 3.4 extends to $F_{\omega}$. As a matter of fact, it follows from the proof, that every finite independent set of realizations of $p_{0}$ in $F_{\omega}$ extends to a basis of $F_{\omega}$. This is not the case for infinite indepedent realizations of $p_{0}$, as we show:

Lemma 3.5: There is an independent set of realizations of $p_{0}$ in $F_{\omega}$, that does not extend to a basis of $F_{\omega}$.

Proof. Let $B=\left\{e_{1} e_{2}^{2}, e_{2} e_{3}^{2}, \ldots, e_{n} e_{n+1}^{2}, \ldots\right\}=\left\{b_{i}: i<\omega\right\}$. Now, because $\left\langle e_{1} e_{2}^{2}, \ldots, e_{n} e_{n+1}^{2}\right.$, $\left.e_{n+1}\right\rangle=F_{n+1}$, we have that $e_{n} e_{n+1}^{2}$ is independent from $\left\{e_{1} e_{2}^{2}, \ldots, e_{n-1} e_{n}^{2}\right\}$. Therefore, $B$ is an independent set of realizations of $p_{0}$.

Claim I. $e_{1} \notin\left\langle b_{i}: i<\omega\right\rangle$.

Proof. Suppose not, then we may assume that $e_{1} \in\left\langle b_{1}, \ldots, b_{n}\right\rangle$, for some $n$. Iterating Fact 3.3 we have $b_{1} b_{2} \ldots b_{n+1}$ is independent from $b_{1}, b_{2}, \ldots, b_{n}$ over $\emptyset$. Thus $b_{1} b_{2} \ldots b_{n+1}$ is independent from $a c l\left(b_{1}, b_{2}, \ldots, b_{n}\right)$ over $\emptyset$. Therefore, $b_{1} b_{2} \ldots b_{n+1}$ is independent from $e_{1}$ over $\emptyset$. But $b_{1} b_{2} \ldots b_{n+1}=e_{1} e_{2}^{3} \ldots e_{n+1}^{3} e_{n+2}^{2}$, so using Fact $3.3 e_{1}^{-1} \cdot e_{1} e_{2}^{3} \ldots e_{n+1}^{3} e_{n+2}^{2}=e_{2}^{3} \ldots e_{n+1}^{3} e_{n+2}^{2}$ is primitive, a contradiction.

Claim II. Let $a \in F_{\omega}$, such that $a \models p_{0}$. Then $\{a\} \cup B$ is a dependent set.

Proof. Suppose not, then $\left\{a, b_{i}: i<\omega\right\}$ is an infinite independent set of realizations of $p_{0}$. We may assume that $a \in F_{n+1}$. Thus, $\left\{a, b_{1}, \ldots, b_{n}\right\}$ is a maximal independent set of realizations of $p_{0}$ in $F_{n+1}$, so, by Theorem 3.4, a basis of $F_{n+1}$. But by our assumption $b_{n+1}$ is independent from $a, b_{1}, \ldots, b_{n}$ over $\emptyset$. Thus, $b_{n+1}$ is independent from $\operatorname{acl}\left(a, b_{1}, \ldots, b_{n}\right)$ over $\emptyset$. Therefore, $b_{n+1}$ is independent from $e_{n+1}$ over $\emptyset$. And $b_{n+1}=e_{n+1} e_{n+2}^{2}$, so using Fact 3.3 $e_{n+1}^{-1} \cdot e_{n+1} e_{n+2}^{2}=e_{n+2}^{2}$ is primitive, a contradiction.

Therefore, $B$ is a maximal independent set of realizations of $p_{0}$ in $F_{\omega}$ that is not a basis of $F_{\omega}$.

Now we get the next easy corollary.

Corollary 3.6: There is $G \prec F_{\omega}$, such that $G$ is not a free factor of $F_{\omega}$.

Proof. By the previous lemma, we only need to show that $G=\left\langle e_{1} e_{2}^{2}, e_{2} e_{3}^{2}, \ldots, e_{n} e_{n+1}^{2}, \ldots\right\rangle=$ $\left\langle b_{i}: i<\omega\right\rangle$ is an elementary substructure of $F_{\omega}$. First note that $t p^{G}\left(e_{1} e_{2}^{2}, e_{2} e_{3}^{2}, \ldots, e_{n} e_{n+1}^{2}, \ldots\right)$ $=t p^{F_{\omega}}\left(e_{1} e_{2}^{2}, e_{2} e_{3}^{2}, \ldots, e_{n} e_{n+1}^{2}, \ldots\right)=p_{0}^{(\omega)}$, this is because $G$ is free with basis $\left\{e_{1} e_{2}^{2}, e_{2} e_{3}^{2}\right.$, $\left.\ldots, e_{n} e_{n+1}^{2}, \ldots\right\}$. Now the proof is straightforward. Let $a_{1}, \ldots, a_{m} \in G, F_{\omega} \models \phi\left(a_{1}, \ldots, a_{m}\right)$ iff $F_{\omega} \models \phi\left(t_{1}\left(b_{1}, \ldots, b_{k}\right), \ldots, t_{m}\left(b_{1}, \ldots, b_{k}\right)\right)$ iff $F_{\omega} \models \psi\left(b_{1}, \ldots, b_{k}\right)$ iff $G \models \psi\left(b_{1}, \ldots, b_{k}\right)$ iff $G \models \phi\left(a_{1}, \ldots, a_{m}\right)$.

We now turn to the main question of the section. That is, whether we can find $a \models p_{0}$ and $\left(b_{i}: i<\omega\right) \models p_{0}^{(\omega)}$ in $F_{\omega}$, such that $a$ forks with each $b_{i}$ over the empty set. As a matter of fact, the next lemma could also serve as an alternative proof of Theorem 3.2 . 
Lemma 3.7: Let $F_{\omega}=\left\langle e_{i}: i<\omega\right\rangle$. Then there exists $\left\{b_{i}: i<\omega\right\}$ an independent set of realization of $p_{0}$ in $F_{\omega}$, such that $e_{1}$ forks with $b_{i}$ over $\emptyset$, for all $i<\omega$.

Proof. Let $B=\left\{e_{1} e_{2}^{2}, e_{1} e_{2}^{3} e_{3}^{2}, e_{1} e_{2}^{3} e_{3}^{3} e_{4}^{2}, \ldots, e_{1} e_{2}^{3} \ldots e_{n}^{3} e_{n+1}^{2}, \ldots\right\}$. Then, $\left\langle e_{1} e_{2}^{2}, e_{1} e_{2}^{3} e_{3}^{2}, e_{1} e_{2}^{3} e_{3}^{3} e_{4}^{2}\right.$, $\left.\ldots, e_{1} e_{2}^{3} \ldots e_{n}^{3} e_{n+1}^{2}, e_{n+1}\right\rangle=F_{n+1}$. Thus, $B$ is an independent set of realizations of $p_{0}$. Furthermore, $e_{1}$ forks with every element of $B$. Suppose not, then $e_{1}$ is independent from $e_{1} e_{2}^{3} \ldots e_{n}^{3} e_{n+1}^{2}$, so, by Fact [3.3] $e_{1}^{-1} e_{1} e_{2}^{3} \ldots e_{n}^{3} e_{n+1}^{2}=e_{2}^{3} \ldots e_{n}^{3} e_{n+1}^{2}$ is primitive, a contradiction.

Now using the invariance of forking, that is whether or not $a$ forks with $b$ over $C$, depends on $\operatorname{tp}(a, b, C)$, we show that $F_{\kappa}$, for $\kappa>\omega$, is not $\aleph_{1}$-homogeneous.

Proposition 3.8: Let $F_{\kappa}=\left\langle e_{i}: i<\kappa\right\rangle$, for some $\kappa>\omega$. Then $F_{\kappa}$ is not $\aleph_{1}-$ homogeneous.

Proof. We first note that, by the proof of Lemma 3.7, $t p\left(e_{1} e_{2}^{2}, e_{1} e_{2}^{3} e_{3}^{2}, e_{1} e_{2}^{3} e_{3}^{3} e_{4}^{2}, \ldots, e_{1} e_{2}^{3} \ldots\right.$ $\left.e_{n}^{3} e_{n+1}^{2}, \ldots\right)=t p\left(e_{1}, e_{2}, e_{3}, \ldots, e_{n}, \ldots\right)=p_{0}^{(\omega)}$. We next show that if we extend $t p\left(e_{1} e_{2}^{2}, e_{1} e_{2}^{3} e_{3}^{2}\right.$, $\left.e_{1} e_{2}^{3} e_{3}^{3} e_{4}^{2}, \ldots, e_{1} e_{2}^{3} \ldots e_{n}^{3} e_{n+1}^{2}, \ldots\right)$ by adding $e_{1}$, then there is no element, $a \in F_{\kappa}$, such that $t p\left(e_{1}, e_{1} e_{2}^{2}, e_{1} e_{2}^{3} e_{3}^{2}, e_{1} e_{2}^{3} e_{3}^{3} e_{4}^{2}, \ldots, e_{1} e_{2}^{3} \ldots e_{n}^{3} e_{n+1}^{2}, \ldots\right)=t p\left(a, e_{1}, e_{2}, e_{3}, \ldots, e_{n}, \ldots\right)$.

Suppose, for the sake of contradiction, that such an element exists. Then there is $n<\omega$, such that $a$ is independent from $e_{n}$ over $\emptyset$ (that is because $a \in \operatorname{acl}(A)$, where $A$ is a finite subset of $\left.\left\{e_{i}: i<\kappa\right\}\right)$. But, by forking invariance, as $t p\left(a, e_{n}\right)=t p\left(e_{1}, e_{1} e_{2}^{3} \ldots e_{n}^{3} e_{n+1}^{2}\right)$, we have that $e_{1}$ is independent from $e_{1} e_{2}^{3} \ldots e_{n}^{3} e_{n+1}^{2}$, which is, as in the proof of Lemma 3.7 a contradiction.

\section{References}

[LS77] R.C. Lyndon and P.E. Schupp, Combinatorial group theory, Springer-Verlag, 1977.

[Mar02] David Marker, Model theory: an introduction, Graduate Texts in Mathematics, vol. 217, Springer, 2002.

[MV03] Armando Martino and Enric Ventura, Examples of retracts in free groups that are not the fixed subgroup of any automorphism, J. of Algebra 269 (2003), 735-747.

[Nie17] Jacob Nielsen, Die isomorphismen der allgemeinen unendliehen gruppe mit zwei erzeugenden, Mathematische Annalen (1917), 385-397.

[Nie03] Andre Nies, Aspects of free groups, J. Algebra 263 (2003), 119-125.

[Per08] Chloé Perin, Elementary embeddings in torsion-free hyperbolic groups, Ph.D. thesis, Université de Caen Basse-Normandie, October 2008.

[Pi196] Anand Pillay, Geometric stability theory, Oxford University Press, 1996.

[Pil08] _ Forking in the free group, J. Inst. Math. Jussieu 7 (2008), 375-389.

[Pil09] _ On genericity and weight in the free group, Proc. Amer. Math.Soc. 137 (2009), 3911-3917.

[Poi83] Bruno Poizat, Groupes stables avec types generiques reguliers, J. of Symbolic Logic 48 (1983), 641-658. 
[Poi01] _ Stable groups, Mathematical Surveys and Monographs, vol. 87, AMS, 2001.

[Sel] Zlil Sela, Diophantine geometry over groups VIII: Stability, preprint, available at http://www.ma.huji.ac.il/ zlil/.

[Sel06] - Diophantine geometry over groups VI: The elementary theory of free groups, Geom. Funct. Anal. 16 (2006), 707-730.

[Sta99] John R. Stallings, Whitehead graphs on handlebodies, Geometric Group Theory Down Under (Canberra 1996), de Gruyter, Berlin, 1999, pp. 317-330.

University of Leeds,

School of Mathematics,

LS2 9JT, UK

rsklinos@maths.leeds.ac.uk 